\title{
Belphégor
}

Littérature populaire et culture médiatique

$17 \mid 2019$

Mutations des légitimités dans les productions culturelles contemporaines

\section{De La Gazette des Études Lupiniennes à L'Aiguille Preuve : 50 ans de bulletins d'érudition populaire}

\section{Cédric Hannedouche}

\section{(2) OpenEdition}

\section{Journals}

Electronic version

URL: https://journals.openedition.org/belphegor/1465

DOI: $10.4000 /$ belphegor.1465

ISSN: 1499-7185

Publisher

LPCM

Electronic reference

Cédric Hannedouche, "de la Gazette des études lupinienNes À l'Aiguille preuve : 50 ANS de BULLETINS D'ÉRUDITION POPULAIRE", Belphégor [Online], 17 | 2019, Online since 16 April 2019,

connection on 25 August 2021. URL: http://journals.openedition.org/belphegor/1465 ; DOI: https:// doi.org/10.4000/belphegor.1465

This text was automatically generated on 25 August 2021.

\section{(i) $\$$}

Belphégor est mis à disposition selon les termes de la Licence Creative Commons Attribution - Pas d'Utilisation Commerciale - Pas de Modification 4.0 International. 


\title{
De La Gazette des Études Lupiniennes à L'Aiguille Preuve : 50 ans de bulletins d'érudition populaire
}

\author{
Cédric Hannedouche
}

1 Le personnage d'Arsène Lupin qu'invente Maurice Leblanc à la demande de Pierre Lafitte au début du $\mathrm{XX}^{\mathrm{e}}$ siècle se révèle un héros à la longévité peu conventionnelle. Encensé par le lecteur en dépit de sa nature de criminel, insaisissable en raison de ses multiples visages, Arsène Lupin a ainsi prospéré durant plus de trente-cinq ans dans les colonnes des feuilletons et des recueils qui recensent ses aventures. Preuve de son passage à la postérité, même après la disparition de Maurice Leblanc, le personnage revient sous la plume d'autres auteurs ${ }^{1}$, passe d'un support à l'autre, différents acteurs l'incarnent à la télévision ou au cinéma ${ }^{2}$. Ce phénomène durable suscite l'intérêt, dans la seconde partie du $\mathrm{XX}^{\mathrm{e}}$ siècle, d'une génération de critiques qui participe à poser les bases d'un regard neuf sur la culture populaire.

2 Ainsi, les premières études lupiniennes s'organisent au tournant des années 1970 dans une revue littéraire qui tire ses origines d'une série de publications mêlant le sérieux à l'absurde. À l'image du héros dont elles révèlent toute la complexité, ces premières études lupiniennes abordent le personnage du gentleman-cambrioleur en tant que production culturelle remarquable.

3 La revue actuelle, L'Aiguille Preuve, apparaît comme l'ultime produit de multiples métamorphoses qui ont conduit à l'élaboration d'une publication semestrielle nourrie d'articles signés d'amateurs séduits par le personnage, qui regroupent aussi bien des lecteurs passionnés que des savants issus de sociétés universitaires et pétris d'une culture scientifique ou des érudits autodidactes faisant preuve d'une connaissance parfaite des aventures d'Arsène Lupin.

4 Elle matérialise l'engagement de l'Association des Amis d'Arsène Lupin. Celle-ci ne dépend d'aucun laboratoire, n'est rattachée à aucune université et ne fait l'objet d'aucun appel à contributions. C'est une revue d'amateurs organisés en association dont l'objectif est de diffuser la meilleure connaissance possible de l'œuvre majeure de 
Maurice Leblanc et de son illustre créature auprès du grand public. Pourtant, son mode de fonctionnement, ses rubriques ou les thèmes retenus évoquent sur bien des points les protocoles de la recherche universitaire. L'étude de son histoire éditoriale, de sa création à nos jours, questionne le statut, le public visé, voire l'identité critique, d'une revue à vocation populaire et donc destinée en apparence au grand public. L'analyse menée ici tentera de démontrer que derrière une légèreté de ton manifeste, les différents bulletins que publient Les Éditions d'Arsène s'inscrivent en définitive dans une approche savante dictée par les patrons de lecture historiquement légués par un groupe d'universitaires.

5 Pour bien saisir les liens qui unissent cette revue à la communauté scientifique, sans doute faut-il ainsi remonter dans un premier temps aux origines des publications lupiniennes, identifier ses membres et ainsi mieux interroger le degré de professionnalisation critique de la revue actuelle. Le rapprochement et la confrontation des différentes générations d'études lupiniennes établit des correspondances évidentes qui invitent au questionnement.

\section{La Revue des Études Lupiniennes}

6 L'Association des Amis d'Arsène Lupin revendique aujourd'hui une filiation qui remonte aux premières études lupiniennes. Celles-ci apparaissent elles-mêmes comme des émanations $d u$ Collège de Pataphysique et d'une volonté d'appliquer rigoureusement un examen sérieux à un sujet situé en-dehors du champ scientifique académique. L'amorce des publications se trouve en effet dans l'exemplaire numéro 2, livré le 10 août 1966, de la revue Subsidia pataphysica [image 1] ${ }^{3}$. Dans cette revue, datée du 29 tatane 93 de l'ère pataphysique ${ }^{4}$, figure une Lettre du Deutérodataire Kirmu au Transcendant Satrape Jean Ferry, Représentant Hypostatique de Sa magnificence Archisphragidophore O.G.G., sur Holmes et Lupin [image 2] ${ }^{5}$. Les deux numéros suivants, du 8 décembre 1967, accueillent un complément du professeur Jean-Claude Dinguirard ${ }^{6}$. Dans son article qu'il intitule "Petite histoire de l'AAAL », Hervé Lechat retrace cette généalogie :

Fort bien documentés et étayés, ces prolégomènes ne tardent pas à susciter des addenda : le numéro suivant des Subsidia ( $n^{\circ} 3 / 4$ du 8 sable 95$)$ accueille Belles Lettres, la réponse circonstanciée de Jean-Claude Dinguirard, éminent enseignant à l'Université du Mirail à Toulouse, qui deviendra Régent de Thermosophie et occupera la chaire de Sagesse Lupinienne (d'aucuns disent que Thermosophie et Lupinologie sont les deux facettes de la même dialectique).? 


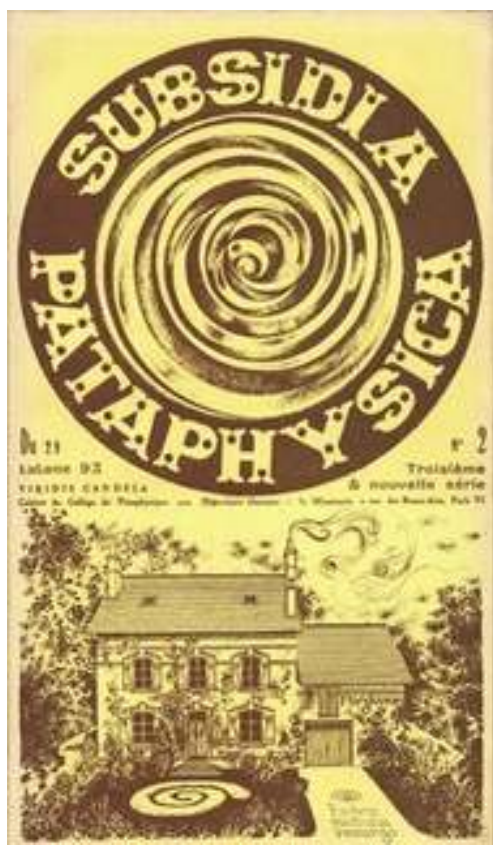

Image 1 : Numéro 2 de la revue Subsidia pataphysica, 10 août 1966

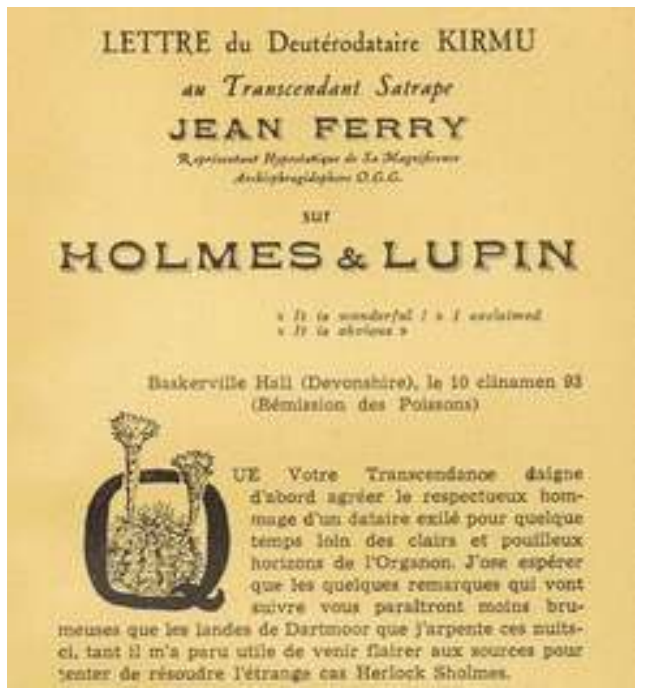

Image 2 : Lettre du Deutérodataire Kirmu au Transcendant Satrape Jean Ferry, Représentant Hypostatique de Sa magnificence Archisphragidophore O.G.G., sur Holmes et Lupin

7 Cette initiative culturelle apparait comme le produit de lecteurs qui entretiennent des liens indiscutables avec la communauté universitaire et qui interrogent de la sorte l'une des nombreuses manifestations de cette «culture de consommation" en construction que décrit Pascal Ory $^{8}$ dans ses travaux. Plusieurs contributeurs de la première revue consacrée au personnage $d$ 'Arsène Lupin se trouvent en effet engagés dans une démarche de réévaluation des productions culturelles en marge des canons légitimés. Ainsi, en 1970, Francis Lacassin ${ }^{9}$ publie une première "Bibliographie de Maurice Leblanc $»^{10}$ qui vise à établir une approche globale des œuvres du père d'Arsène Lupin. Cette démarche neuve consonne avec celle que le fondateur du Club des bandes dessinées avait entamé quelques années plus tôt lorsqu'il avait mis en lumière des fonctionnements culturels majeurs par l'intermédiaire d'un périodique ${ }^{11}$. 
Dans les deux cas, il convient de remarquer que la critique nouvelle émane systématiquement d'une structure externalisée des pôles de recherches universitaires.

Depuis sa création, l'Association des Amis d'Arsène Lupin prolonge l'humour et la désinvolture des publications originelles que l'on trouvait dans les pages de la Revue des Études Lupiniennes. Dans son article «Un lustre d'études lupiniennes » ${ }^{12}$, Jean-Claude Dinguirard revient sur la création et la mission de la Revue des Études Lupiniennes : « la R.E.L. se présente comme "l'organe de la Société des Etudes Lupiniennes" (S.E.L. en abrégé). D'après les informations que l'on peut glaner au fil des pages, cette S.E.L. - qui n'a fait l'objet d'aucune déclaration de naissance, d'aucun dépôt de statuts auprès des organismes compétents - se propose, comme son nom l'indique, d'explorer le mythe d'Arsène Lupin $»^{13}$. Le style impersonnel qu'emprunte ici l'auteur dans son article évoque celui de l'observateur impartial qui impose une distance entre l'objet et son jugement. Il convient néanmoins de souligner que c'est Jean-Claude Dinguirard luimême qui est à l'origine de la S.E.L. quand il se pare « [...] dans une lettre à l'auteur d'un article sur Lupin, du titre de directeur de la SEL ${ }^{14}$ et qu'il se dissimule derrière les articles signés sous les pseudonymes de Anqueti-Turet, Perry Hammer ou encore Maurice Dubourg. De son côté, Paul Gayot rappelle que

[1]es mots, Revue des Études Lupiniennes apparaissent pour la première fois dans le numéro 3-4 des Subsidia Pataphysica (sable 95-décembre 1967 vulg.). C'était à l'occasion d'une précision apportée par Jean-Claude Dinguirard à une étude de K. Kirmu publiée dans le précédent numéro des dits Subsidia (Lettre au T.S. Jean Ferry sur Holmes et Lupin). ${ }^{15}$

9 Jean-Claude Dinguirard se positionne de la sorte au cœur d'un dispositif parodique de recherche littéraire. Il est tout à la fois acteur et observateur de la production critique à l'origine des études lupiniennes. La bibliographie ${ }^{16}$ que propose l'Association des Amis d'Arsène Lupin mentionne également l'existence, entre 1965 et 1966, de quatre numéros de La Gazette des Études Lupiniennes ainsi que la publication d'un Bulletin de la Société fermière des Études Lupiniennes au cours des années 1967 et $1968^{17}$.

La naissance de la Société des Études Lupiniennes (la S.E.L.), n'a fait l'objet d'aucune déclaration, comme on l'a dit ; elle relève de la création inattendue et spontanée par un public lupinophile. Jean-Claude Dinguirard, qui en est l'une des chevilles ouvrières, note dans l'article qu'il lui consacre que «[s]elon des sources bien informées, il existerait de par le monde moins de vingt collections complètes de la Revue des Études Lupiniennes ». Celle-ci, que ses éditeurs présentent alors comme un "fanzine », est lancée en 1967 avec d'emblée un numéro 5 (image 3) (« 24 pages, recto seul. Papier citron, couverture rose. Titre : “R.E.L. 5" ms. Pas de justification de tirage »). Cette date marque très clairement l'avènement et l'organisation d'un fandom lupinien, clairement initié par une communauté scientifique qui se désengage de la sphère de recherche traditionnelle pour promouvoir un objet culturel hors du champ académique. La revue est distribuée gratuitement sur demande. 


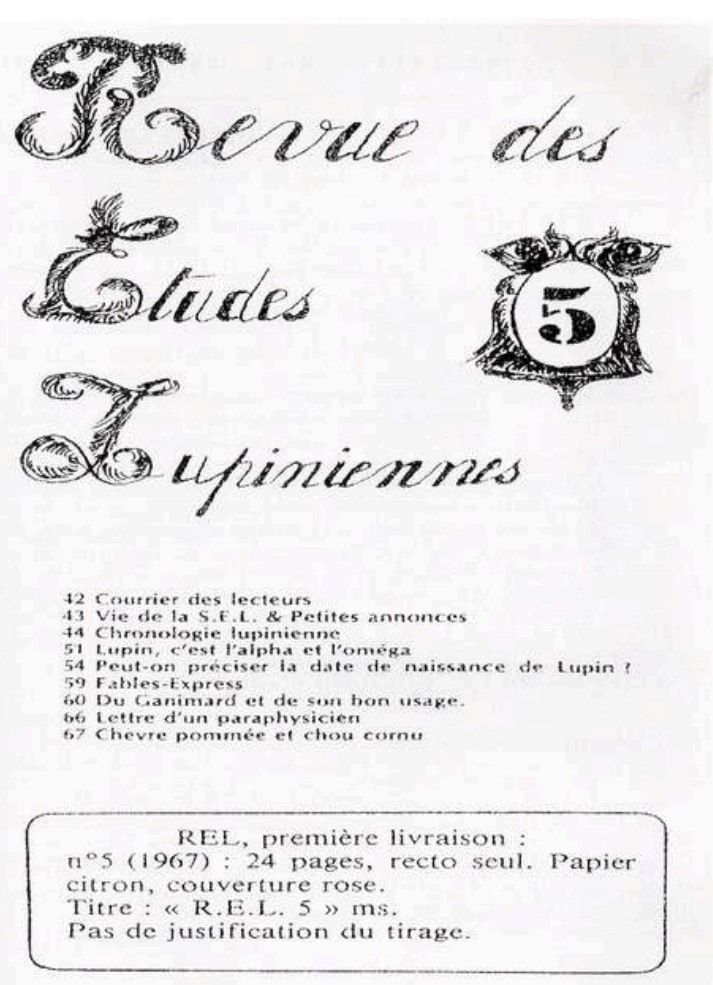

Image 3 : Numéro 1 référencé numéro 5 de la Revue des Etudes Lupiniennes, 1967

11 À la suite $d u n^{\circ} 5$, paraît le $n^{\circ} 4$ (image 4 ) : «26 pages, recto seul. Papier blanc, couverture ill. (dessin coul. Signé Gil), l'ill. h.t. dans les exemplaires de tête. Justification de tirage: "32 ex. (de luxe), savoir 4 ex. canins sur verge candide, 8 ex. incisifs sur velin blanc acide, 20 ex. mâcheliers sur grand émail, un peu cariés, l'ensemble constituant l'édition originale" ${ }^{18}$. Ce numéro parait en 1968, le 3 en 1969 (image 5) : « 32 pages, recto-verso. Papier jaune d'or, couverture ill. (dessin coul. Signé Karslberg), l'ill. dans le texte, l'ill. hors texte, 1 publicité "La R.E.L. cite ses sources : Désiré (3), Subsidia Pataphysica (4), Via Domitia (5)". Justification de tirage: "17 exemplaires d'un luxe criard et 35 ex. sur papier de Médiocrité, Doré $»^{19}$, le numéro 2 en 1970 (image 6) : « 37 pages, recto-verso. Papier jaune paille et saumon (pp. 25-37). Couverture ill. faisant sommaire, jaquette signée G.P. Paris, 1 ill. h.t. Justification de tirage : "15 ex. nominatifs ont été tirés, avec un luxe sardanapalesque, pour les amis et bienfaiteurs de la R.E.L. $»^{20}$. Deux autres numéros paraissent en $1970:$ " $\mathrm{n}^{\circ} 1$ et 0 ensemble (1970) (Les deux volumes de cette livraison sont connus, en conséquence, sous le nom de " $\left.\mathrm{n}^{\circ} 10^{\prime}\right): 61$ pages, recto-verso. Papier de teintes diverses $\left(\mathrm{n}^{\circ} 1: \mathrm{pp}\right.$. I-III, 7-16, 19-20: saumon ; pp. 1-6, 17-18 : bleuâtre ; pp. 21-32 : jaune - nº : pp. 1-17 : bleu vif; pp. 17-19: saumon). Couverture du $n^{\circ} 1$ ill. (dessin agrémenté d'objets divers, le tout signé Hib) [image 7] ; Couverture du n 0 typographiée [image 8]; en tout 5 ill. intérieures. Justification de tirage : "Il a été tiré plusieurs exemplaires, quelques-uns pour les amis et bienfaiteurs de la R.E.L.; d'autres, moins exquisément ploutocratiques et élitéens, pour le commun des lupinophiles" $»^{21}$. 


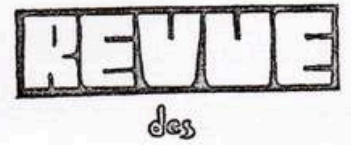

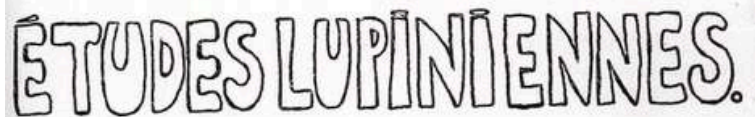

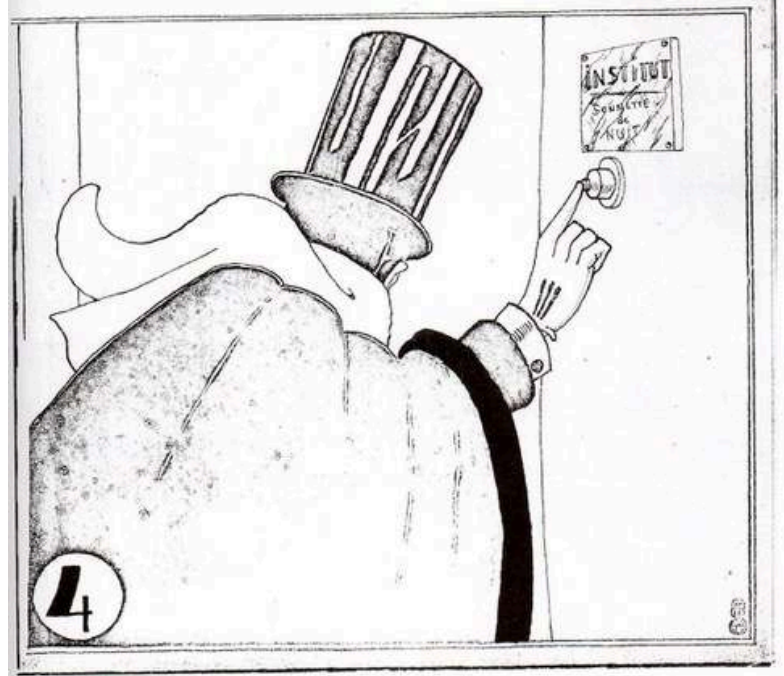

Image 4 : Numéro 2 référencé numéro 4 de la Revue des Etudes Lupiniennes, 1968
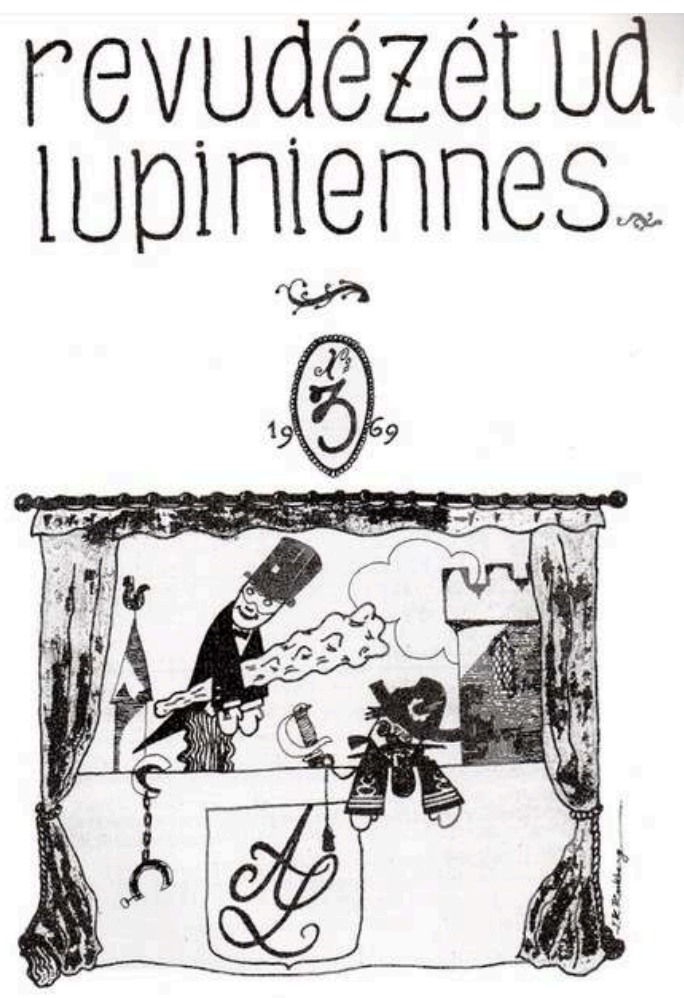

Image 5 : Numéro 3 référencé de la Revue des Etudes Lupiniennes, 1969 


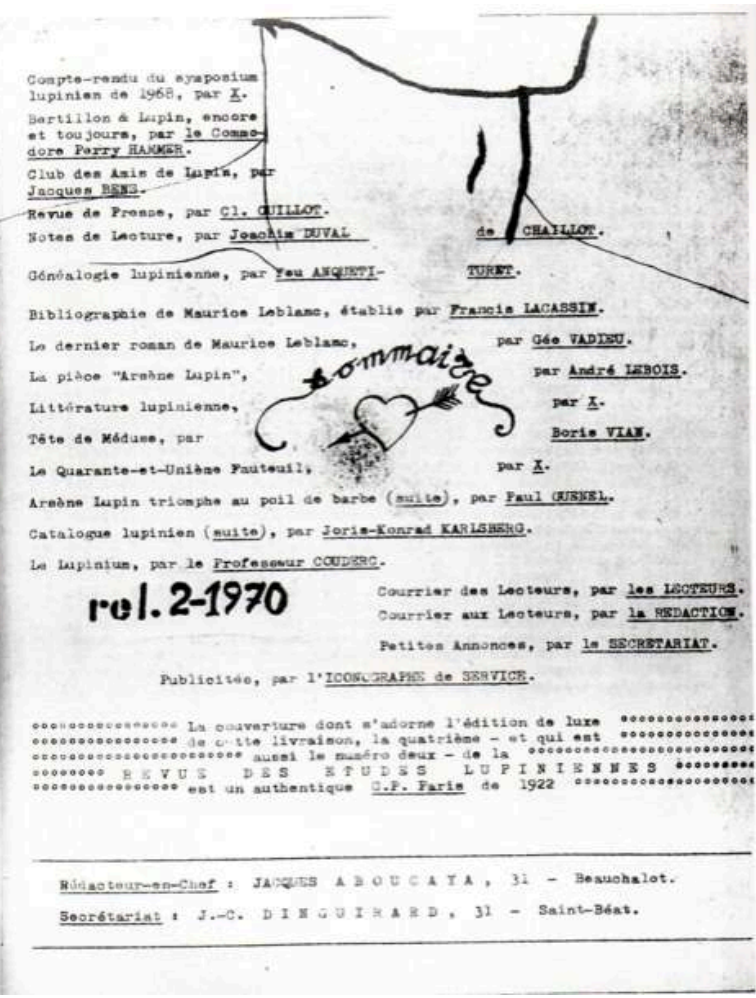

Image 6 : Numéro 4 référencé numéro 2 de la Revue des Etudes Lupiniennes, 1970

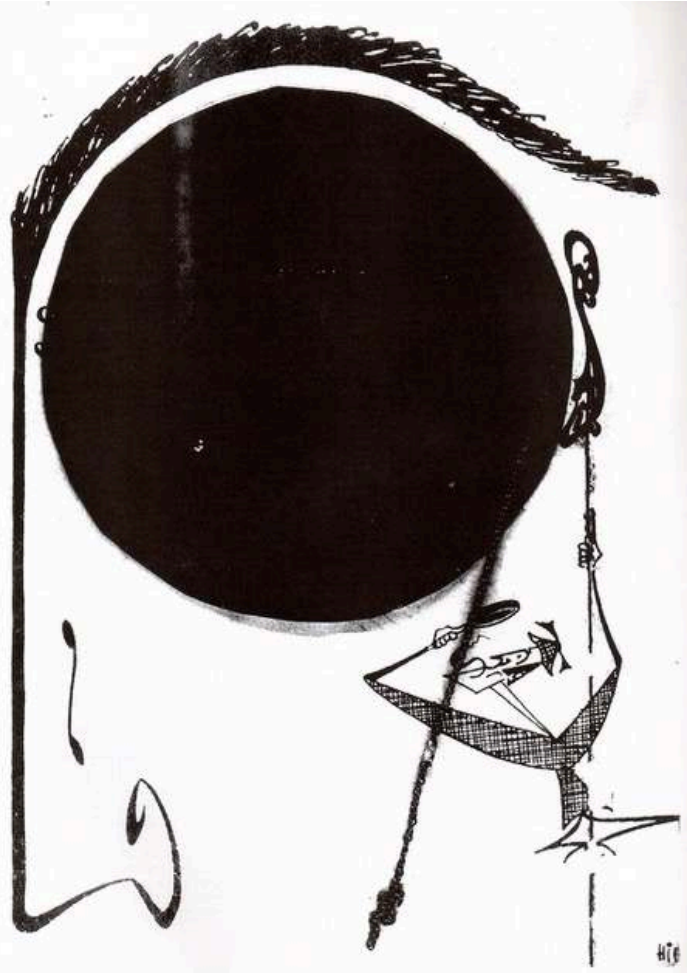

Image 7 : Numéro 5 référencé numéro 1 de la Revue des Etudes Lupiniennes, 1970 


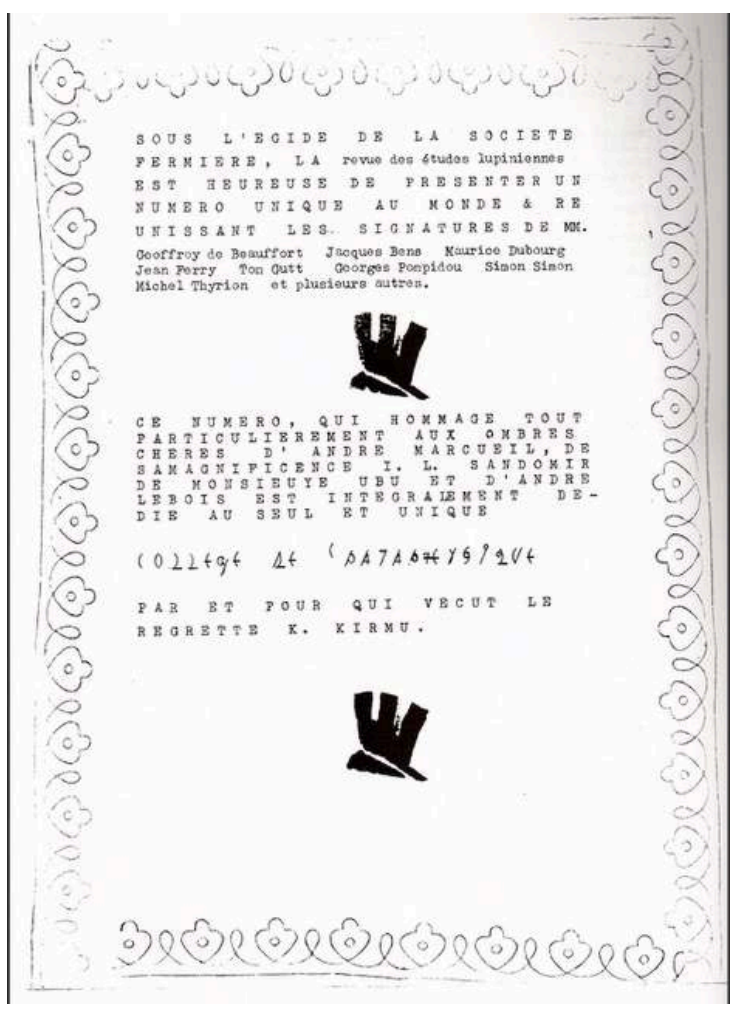

Image 8: Numéro 6 référencé numéro 0 de la Revue des Etudes Lupiniennes, 1970

Ce classement à rebours initié par la parution du numéro 5 en 1967 dénote d'emblée le projet éphémère de ses auteurs. Cette programmation initiale du terminus ad quem incarné par le double numéro de 1970 suggère une volonté de réunir dans une temporalité réduite et sous une même bannière des auteurs aptes à conduire une entreprise scientifique. Cette revue «se déclare alors éditée par la Société Fermière de la S.E.L. et des allusions discrètes sont faites à une Commission Inquisitoriale Acroamatique (C.I.A. en abrégé) $»^{22}$.

Chaque livraison comprend des rubriques de «Courrier des lecteurs» (un «Courrier des Lecteurs, par les Lecteurs», un "Courrier aux Lecteurs, par la Rédaction»), de "varia », des poèmes, une rubrique sur la "Vie de la Société des Études Lupiniennes » et d'autres rubriques de nature hétérogène comme un "Compte-rendu du symposium lupinien du 30 octobre 1968 (C.I.A.-S.E.L.) »"23. La R.E.L. publie également les poèmes de ses collaborateurs comme les « Fables-Express $»^{24}$ de Jacques Aboucaya.

Les articles qui les composent alors apparaissent, avant tout, comme des jeux d'esprit mêlant des interrogations ainsi que des mises au point nécessaires, comme un "Catalogue des pseudonymes et identités lupiniennes $»^{25}$ assez exhaustif puisque composé de 124 entrées, un autre sur "D’Artagnan et Lupin » ${ }^{26}$, ou sur "Les sources balzaciennes d'Arsène Lupin $»^{27}$. En 1968, un article s'interroge sur "Trois incarnations d'Arsène Lupin au cinéma » et un autre s'intitule «Symbolique et Sexualité dans les Aventures d'Arsène Lupin ». La revue s'intéresse tout autant à Maurice Leblanc qu'à son personnage. Certaines pages ne sont même pas référencées dans les sommaires et soulignent l'intention rédactionnelle, liée à l'objet d'étude, de distance avec les conventions. Ainsi, une première tentative de «Filmographie lupinienne » apparaît à la page 13 du numéro 3 de la R.E.L. sans être annoncée au seuil de l'exemplaire. 
15 A côté de ces articles aux incontestables vertus scientifiques, le lecteur circule dans un déchaînement de points de vue appuyés sur l'absurde. L'humour, la parodie, le grotesque sont alors omniprésents: «De l'influence du manichéisme cathare sur le mécanisme de la pensée inductive chez l'inspecteur Ganimard ", " Arsène Lupin faisaitil de l'aérophagie? $»^{28}$, «Les pyjamas de Lupin $»^{29}$, "Essai d'analyse spectrale du discours lupinien $»^{30}$, ou encore "Une source lupinienne négligée : Nostradamus». Cependant, toutes ces productions font preuve d'une précision reconnue et d'une grande connaissance des aventures d'Arsène Lupin dans leurs citations. La volonté scientifique s'affirme ainsi au-delà d'une apparente forme de plaisanterie. L'utile rejoint nécessairement l'agréable en sollicitant de façon constante l'esprit et la sagacité du lecteur.

Les articles sont généralement anonymes, signés d'un pseudonyme (Géo Vadieu, Hercule Papy ou Ingmar d'Ainjust, anagramme de Justin Ganimard ${ }^{31}$ ). Les collaborateurs de la R.E.L. viennent de différents horizons : des amoureux de littérature populaire, souvent policière ou de Maurice Leblanc, comme Michel Lebrun, et du surréalisme (René Alleau, Tom Gutt). L'Oulipopo ${ }^{32}$ y participe activement ainsi que des membres du collège de pataphysique. Francis Lacassin y poursuit son entreprise de diffusion d'une littérature des marges et des cultures populaires. Jean-Claude Dinguirard justifie la collusion de cette diversité d'esprits et de plumes de la façon suivante :

Nous croyons plutôt discerner dans cet enthousiasme d'esprits venus d'horizons si divers, pour une revue qui ne leur apporterait ni profit matériel, ni gloire, la promesse d'une proche reconnaissance officielle d'un personnage qui mérite mieux : Arsène Lupin..$^{33}$

17 Pour Jacques Rolland de Renéville ${ }^{34}$, un autre membre contributeur de la R.E.L., une telle reconnaissance devait inévitablement passer par la reconnaissance universitaire : le but à atteindre pour lui, c'était le dépôt et la soutenance d'une thèse consacrée à Arsène Lupin. Le doyen de l'université d'Abidjan, comme le rapporte ici Jean-Claude Dinguirard, avait à l'époque invité Michel Lebrun ${ }^{35}$ à poursuivre son travail de recherches qui consistait à démontrer la reprise chez d'autres auteurs des «traits pertinents » présents déjà chez Lupin pour créer de nouveaux personnages comme « Le Saint » faisant ainsi du personnage un archétype et un modèle culturel.

Ces Eléments... de Michel Lebrun auraient mérité une diffusion bien supérieure. Dans ce cas au moins, tout espoir n'est pas perdu : nous ne croyons pas trahir un secret en révélant que J. Rolland de Renéville, Doyen de la Faculté des Lettres d'Abidjan, presse Michel Lebrun de transformer ses «Eléments...» en Thèse de $3^{\mathrm{e}}$ cycle... L'ouvrage deviendrait ainsi accessible aux chercheurs, à qui il rendrait service, en mettant à leur disposition une masse de renseignements d'un grand intérêt. ${ }^{36}$

18 En moins de cinq ans, cette revue d'amateurs éclairés aura balisé bien des angles de la recherche universitaire en littérature populaire. Travaillant à la fois sur les aventures d'Arsène Lupin, ses sources et ses réalisations transmédiatiques, les articles qui composent la Revue des Études Lupiniennes démontrent la capacité à interroger un texte situé en-dehors du canon des anthologies littéraires et de la tradition. Après ces numéros, la publication critique consacrée au personnage d'Arsène Lupin semble s'éteindre. Il convient cependant de mentionner que la revue Enigmatika ${ }^{37}$ consacre ses numéros 2 (1976) [image 9] et 8 (1978) au gentleman-cambrioleur, tout comme le numéro de Europe d'août et septembre 1979. 


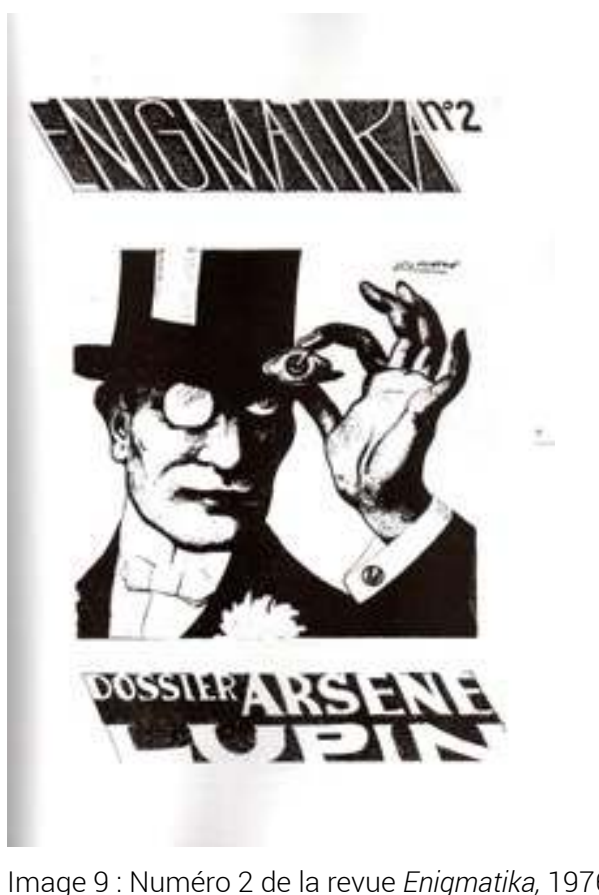

\section{Nouvelles vies des études lupiniennes}

La naissance de l'Association des Amis d'Arsène Lupin remonte à 1985. Précisément, les statuts en ont été officiellement déposés en juin 1985 avec une première Assemblée Générale en 1986. La paternité de l'Association revient au philosophe et essayiste François George. Il s'agit dès l'origine d'une association indépendante régie par la loi de 1901. Comme son nom l'indique, cette association d'amateurs ne se tourne pas vers l'hagiographie exclusive de l'auteur Maurice Leblanc. Elle se réclame avant tout du personnage phare de l'auteur normand: Arsène Lupin. Ses membres privilégient dès lors les impressions de lecture des aventures du gentleman-cambrioleur, et la revue devient l'épicentre du fonctionnement de l'association, qui revendique son attachement à la ville d'Étretat. Les discours des assemblées y sont transcrits, les études se cantonnent aux approches morales, philosophiques et psychanalytiques et révoquent les particularités littéraires ou stylistiques.

A l'époque de sa création, au milieu des années 1980 donc, les études reposent sur des approches essentiellement phénoménologiques. François George ${ }^{38}$ 'entoure de ses amis intellectuels et anciens professeurs, et de quelques membres de la S.E.L. : André ComteSponville, Valère Catogan (pseudonyme derrière lequel se cache Raymond Lindon), ou encore Jacques Rolland de Renéville. On y croise aussi les noms de Jacques BourbonBusset, de l'Académie française, le cruciverbiste Robert Scipion ou encore Claude Aveline. Des conférences sont dès ce moment organisées, une nouvelle revue est lancée - c'est L'Aiguille creuse, à la courte destinée. Surtout, l'association centralise les documents lupiniens à Étretat et peut compter sur un fonds vertigineux. Des conclaves et rassemblements sont mis en place. Sous cette ère, l'œuvre de Maurice Leblanc est notamment abordée sous l'angle de la morale, de l'éthique et de la psychanalyse. Une fois encore, l'action critique ne dure pas et à partir de 1990 les publications s'arrêtent. 
21 En 2004 enfin, c'est une nouvelle revue qui est lancée, L'Aiguille Preuve, avec un statut clairement défini par la loi. La rupture avec les conclaves et la vision de la décennie précédente est affichée. Les membres de l'Association, tout comme ceux de la S.E.L. des temps passés, ne présentent pas a priori un profil homogène. On y trouve comme à l'origine des amoureux lupiniens souhaitant renouer avec leurs lectures de jeunesse, des lecteurs de romans policiers Belle Époque, des holmésiens, certains passionnés de mystères ésotériques comme celui de Rennes le Château, comme des amateurs de l'histoire normande et de ses falaises. Cette fois, la forme de la revue emprunte ses codes à ceux de la recherche universitaire, même si les auteurs recouvrent des origines radicalement différentes. Le président de l'Association, Hervé Lechat, signale entre autres :

un ancien major de l'armée de terre, un ancien directeur des services d'une ville côtière normande, un adjoint au maire d'Etretat, un ingénieur électronicien qui a formé des générations de jeunes bricoleurs de circuits et de puces, un biologiste, deux ou trois profs de lycée, deux journalistes, un ingénieur EDF, une adjointe de sécurité à Orly, un retraité de banque, un ouvrier syndicaliste de la métallurgie, un propriétaire de vignobles en Champagne, le troisième plus grand vendeurs de livres en France (Michel Bussi), un professeur d'odontologie de la Loma Linda University of South California, des habitants d'Etretat, un industriel belge, un Corse, deux Italiens.

Ses membres historiques encore présents sont : Robert Jaffray, l'acteur Bernard Lavalette (qui joua dans les séries télé avec Georges Descrières et dont le grand-père Maurice de Fleury était intime avec Georgette et Maurice Leblanc), Philippe Radé, un général d'aviation à la retraite fils du photographe des expéditions himalayennes Marcel Ichac. Notons que Georges Descrières, Jean-Claude Brialy, Jean-Noël Jeanneney, Claude Mauriac ont fait partie de l'Association (souvent à titre honorifique). Le nombre de membres oscille depuis entre 40 et 80 avec un noyau dur d'une trentaine de permanents. L'Association des Amis d'Arsène Lupin, en tant qu'association d'amateurs, demeure très fragile. Aujourd'hui, elle repose essentiellement sur des protocoles proches de ceux employés par les revues universitaires. Deux publications par an, au printemps et à l'automne, une liste de diffusion régulière, une "veille lupinienne ", ainsi qu'un bulletin informatique, L'Écho de France, qui reprend et annonce les expositions, les salons et les publications autour d'Arsène Lupin et de Maurice Leblanc. L'assemblée générale est organisée à la fin du mois de mai de chaque année. Des conférences publiques y sont données, le bilan de l'association est dressé.

L'Aiguille Preuve paraît aux Éditions d'Arsène, subventionnées par la ville d'Étretat et la région au nom du patrimoine. L'ouvrage au format A4 compte généralement une centaine de pages avec illustrations et textes sur deux colonnes. Le volume soigné se veut important, loin de la feuille de publication sommaire. Le contenu de la revue perpétue la tradition pataphysique des origines avec des articles qui présentent encore des raisonnements fondés sur l'absurde qui sont souvent l'œuvre d'auteurs possédant une excellente maîtrise des aventures du gentleman-cambrioleur. Des moments d'humour et de dérision allègent ainsi un volume qui refuse l'esprit trop sérieux. $\mathrm{Ne}$ revendiquant aucune mission de recherche, quelques auteurs usent encore de pseudonymes, noms de personnages ou d'anagrammes : l'abbé nédictin, René Pulsani, Pr Gerbois, Capitaine Jeanniot, Hortense Daniel. Le ton exprime une légèreté et une volonté de divertir son lecteur. Cette particularité tonale apparaît particulièrement dans la rubrique «Lupinerie », ultime vestige de la tradition pataphysique. 

de découverte ou de redécouverte du champ lupinien. Contrairement à la génération de François George, les collaborateurs de L'Aiguille Preuve ne délaissent pas le père d'Arsène Lupin et intègrent Maurice Leblanc dans leurs questionnements. Descendante lointaine de La Revue des Études Lupiniennes, L'Aiguille Preuve rejoint peu à peu les attentes d'une revue de recherche scientifique. Depuis 2012, chaque numéro s'efforce de paraître avec une thématique spécifique, comme celui de 2014 consacré à la Grande Guerre ou un autre sur la réception internationale d'Arsène Lupin: "Arsène Lupin, hors-les-murs ». Le sommaire comprend désormais une rubrique "Textes retrouvés " où sont réédités des interviews, des articles sur Maurice Leblanc, des textes inédits, une rubrique «Études lupiniennes ». Là, le comité de rédaction fait le choix de reproduire des articles à valeur scientifique traitant du monde lupinien. On trouve ainsi des extraits de thèse («Pour en finir avec Jacob» de Jean-Marc Delpech ${ }^{39}$ ) et des publications, comme celle de Daniel Aranda «Maurice Leblanc et la résurgence de la série $»^{40}$, "L'Étrange Voyage $»^{41}$ de Michel Bussi, un autre article de Hubert Duval intitulé «Jean Jaurès chez Arsène Lupin ${ }^{42}$, d'autres de Paul Gayot ou de Marc Lits, "Lupin ou la diffraction transmédiatique " ${ }^{43}$.

Certains articles signés des amateurs de l'Association vont même jusqu'à corriger les imperfections des autorités antérieurement admises. Aussi, dans son article «La Vraie vie d'Arsène Lupin ou les Mille-et-une erreurs de Francis Lacassin ${ }^{44}$, Philippe Radé reprend la biographie fictive que retrace Francis Lacassin pour la publication des aventures d'Arsène Lupin chez Robert Laffont en 1986 (tome 3) afin de l'amender, la revoir et la compléter. Celle-ci sera à nouveau absorbée et largement complétée par le numéro 18 bis d'octobre 2016 dans l'article "Chronolupin » signé René Pulsani qui reprend et intègre chaque récit du personnage. Ce dernier commence son travail avec la date du 25 juillet de l'année 732 avant notre ère pour la terminer le 6 novembre 1941 à la mort de Maurice Leblanc.

26 La rubrique " Géographie lupinienne » s'attache, de son côté, à revenir sur les lieux qui ont pu inspirer Maurice Leblanc qui dans ses romans n'a de cesse de reconfigurer le réel, de le travestir au moyen d'une toponymie imaginaire. Il est clair que la revue actuelle profite assez nettement de l'acceptation des cultures populaires dans les nouveaux champs universitaires. De cette manière, le lecteur assiste à une régularisation des sommaires qui dupliquent ceux des bulletins critiques traitant des anthologies littéraires. Même si le lecteur de L'Aiguille Preuve retrouve les procédés d'écriture et rédactionnels utilisés autrefois par les pataphysiciens, il apparaît que ces derniers relèvent désormais davantage de l'hommage et de l'affirmation chez les membres de l'association d'une paternité assumée, et donc révérée, pour les intuitions des pionniers.

En conclusion, une telle revue centralise les découvertes, multiplie les hypothèses et propose des études approfondies des textes de Maurice Leblanc tout en gardant un esprit de légèreté, d'humour, sur lequel elle a été fondée. Elle cite, est à son tour citée, et obtient de la sorte droit de cité dans la recherche littéraire. En dépit de sa faible notoriété, elle assume une fonction de chronique scientifique centrale en littérature populaire. Elle rend compte, elle témoigne d'une actualité vivante sans pour autant prétendre à un rôle de recherche prospective. De façon évidente, les mutations de cette revue balisent et suivent les étapes d'une recherche en littérature populaire et policière en France depuis les années 1970. Elles démontrent l'importance d'une critique qui se 
développe en marge des circuits traditionnels en littérature et qui tend peu à peu à s'institutionnaliser à partir du moment où l'objet d'étude obtient de son côté une forme de reconnaissance universitaire.

\section{NOTES}

1. Citons par exemple Boileau-Narcejac (Le Secret d'Eunerville, La Poudrière, Le Second visage d'Arsène Lupin, La Justice d'Arsène Lupin, Le Serment d'Arsène Lupin,1973-1979) et Michel Zink (Arsène Lupin et le mystère d'Arsonval, 2004) dont les romans remettent en scène le célèbre personnage.

2. Parmi les acteurs qui ont prêté leurs traits à Arsène Lupin à l'écran, on se souvient entre autres de John Barrymore (Arsène Lupin, 1932), de Robert Lamoureux (Les Aventures d'Arsène Lupin, 1957 et Signé Arsène Lupin, 1959) et de Romain Duris (Arsène Lupin, 2004) pour le cinéma ainsi que de Georges Descrières (Arsène Lupin, 1971-1974), Jean-Claude Brialy (Arsène Lupin joue et perd, 1980) ou François Dunoyer (Le Retour d'Arsène Lupin, 1989-1990, Les Nouveaux exploits d'Arsène Lupin, 1994-1995) pour la télévision.

3. Subsidia pataphysica est le titre donné à la revue du Collège de Pataphysique. Placée sous la direction d'Emmanuel Peillet, elle compte vingt-huit numéros publiés entre 1965 et 1975. On y retrouve des interventions de Paul Gayot, Georges Perec, Eugène Ionesco ou Raymond Queneau.

4. L'ère pataphysique commence officiellement le 8 septembre 1873 avec la naissance d'Alfred Jarry.

5. La lettre est datée du 10 Clinamen 93.

6. Jean-Claude Dinguirard (1940-1983) fut professeur à l'Université de Toulouse II. Reconnu pour ses travaux en sciences du langage et pour sa thèse intitulée Ethnolinguistique de la haute vallée du Ger soutenue en 1975, Jean-Claude Dinguirard est considéré comme l'un des premiers chefs de file d'une réflexion critique autour du personnage d'Arsène Lupin.

7. L'article sous-titré «L'AAAL, son histoire, ses mythes et ses légendes » se trouve intégralement reproduit dans le numéro 19 bis d'octobre 2017 de L'Aiguille Preuve (p. 2-4). Son auteur, Hervé Lechat, s'y efforce de clarifier l'histoire de l'association qu'il préside désormais depuis 2014.

8. Auteur d'une thèse de doctorat soutenue en 1990, Pascal Ory, lui-même Régent du Collège de Pataphysique, propose de réfléchir aux politiques culturelles françaises et leurs conséquences depuis le Front Populaire.

9. Francis Lacassin (1931-2008) fut l'un des premiers spécialistes des productions populaires. Il fonde en 1962 le Club des bandes dessinées et préface de nombreux ouvrages dans la collection «Bouquins » des Éditions Robert Laffont.

10. Dans le numéro 2 de la Revue des Études Lupiniennes (p. 13-25).

11. Publié de juillet 1962 à mars 1967, le magazine Giff-Wiff est placé sous la direction de Francis Lacassin.

12. L'article « Un lustre d'études lupiniennes » de Jean-Claude Dinguirard paraît pour la première fois dans la revue Littératures XIX en 1972 (p. 103-110). Il sera ensuite reproduit dans le numéro 2 de la revue Enigmatika (p. 5-12) ainsi que dans le numéro 19 bis de L'Aiguille Preuve (octobre 2017, p. 10-17).

13. Jean-Claude Dinguirard, « Un lustre d'études lupiniennes », Littératures XIX, 1972, p. 103.

14. Jacques Aboucaya, « Hommage à J.-C. Dinguirard », L'Aiguille Preuve, $\mathrm{n}^{\circ} 19$ bis, p. 7.

15. Paul Gayot, «Précision thermosophique ». 
16. L'Association des Amis d'Arsène Lupin propose régulièrement une mise à jour bibliographique qu'elle intègre dans chaque numéro de L'Aiguille Preuve.

17. Dans L'Aiguille Preuve, numéro 19 bis, 2017, p. 215 à 219.

18. Jean-Claude Dinguirard, « Un lustre d'études lupiniennes », art. cit., p. 103.

19. Ibid.

20. Ibid.

21. Ibid.

22. Ibid.

23. Tous ces exemples sont tirés du n ${ }^{\circ} 2$ de la R.E.L. (1970).

24. R.E.L., numéro 5, 1967.

25. Anqueti-Turet, R.E.L., numéro 4, 1968.

26. Patrice Gauthier, R.E.L., numéro 10, 1970.

27. Dubourg Maurice, R.E.L., ibid.

28. Professeur Couderc, R.E.L., ibid.

29. Géo Vadieu, R.E.L., numéro 3, 1969.

30. Jacques Aboucaya, R.E.L., numéro 3, 1969.

31. Célèbre policier inventé par Maurice Leblanc pour traquer le gentleman-cambrioleur.

32. L'Oulipopo (Ouvroir de littérature policière potentielle) fut créée en 1973 par le pataphysicien François le Lionnais sur le modèle de l'Oulipo. Véritable groupe de recherche, l'Oulipopo se propose d'étudier et de recenser les mécanismes et situations du roman policier d'énigme.

33. Jean-Claude Dinguirard, « Un lustre d'études lupiniennes », art cit., p. 110.

34. L'universitaire Jacques Rolland de Renéville est notamment l'auteur des articles « Devinette littéraire » et « Lettre » parus dans le numéro 10 de la R.E.L. (1970).

35. Parallèlement à sa colossale production romanesque, Michel Lebrun (1930-1996) fut un membre fondateur de l'Oulipopo. Il est notamment l'auteur d'un travail intitulé Eléments pour une étude comparative des imitateurs, émules et épigones d'Arsène Lupin (repris dans le numéro 604 que la revue Europe consacre à Arsène Lupin en 1979) qui n'aboutira pas à la thèse de doctorat souhaitée par Jacques Rolland de Renéville.

36. Jean-Claude Dinguirard, «Un lustre d'études lupiniennes ». L'article est présent dans le numéro 2 de la revue Enigmatika en 1976. La confidence relatée remonte à ces années.

37. Enigmatika paraît pour la première fois en février 1976. Il s'agit du bulletin qui publie les différents articles du groupe de l'Oulipopo.

38. François George publie en 1978 un ouvrage intitulé La Loi et le Phénomène. Les preuves de l'existence d'Arsène Lupin.

39. L'Aiguille Preuve, $\mathrm{n}^{\circ} 14,2012$.

40. L'Aiguille Preuve, $\mathrm{n}^{\circ} 12,2010$.

41. Ibid.

42. Ibid.

43. L'Aiguille Preuve, $\mathrm{n}^{\circ} 10,2008$.

44. L'Aiguille Preuve, n6, 2004. 


\section{ABSTRACTS}

Fondée en 1985 par le philosophe et essayiste François George, l'Association des Amis d'Arsène Lupin se présente comme une association littéraire indépendante régie par la loi de 1901. Elle compte parmi ses adhérents aussi bien des amateurs des aventures du gentleman-cambrioleur que des membres proches de la communauté scientifique dont plusieurs spécialistes et biographes de Maurice Leblanc. Elle est à l'origine de deux publications : un bulletin diffusé en ligne (L'Écho de France) et L'Aiguille Preuve (aux Éditions d'Arsène), sa principale publication, d'abord annuelle puis semestrielle depuis 2014. L'Aiguille Preuve prolonge une littérature d'amateurs qu'initie La Gazette des Études Lupiniennes dès 1965.

Elle répertorie aujourd'hui les dernières avancées universitaires consacrées au personnage tout en proposant des textes retrouvés ou réédités et des fantaisies d'amateurs éclairés.

\section{INDEX}

Mots-clés: Maurice Leblanc, roman populaire, édition, culture médiatique, presse

\section{AUTHOR}

\section{CÉDRIC HANNEDOUCHE}

Université d'Artois, Arras, Laboratoire Textes et Cultures, EA 4028 